\title{
Analysis and Application of Hyperspectral Image Data of Raw Egg Yolk
}

\author{
Shiqi Huang, Wensheng Wu, Yuting Zhang, Xiangyang Duan \\ School of Information Engineering, Xijing University, Xi'an, 710123 China
}

Keywords: Raw yolk; Hyperspectral image; Egg quality; Spectral characteristics

\begin{abstract}
Using new technical means to effectively identify the quality of eggs is a common concern of people and the supervision and management department. This paper uses the advanced hyperspectral remote sensing imaging technology to study the spectral characteristics of egg yolk. The hyperspectral image data of egg yolk is obtained, spectral characteristic data is extracted from hyperspectral image data, spectral characteristics of different types eggs is analyzed, and finally, the quality of eggs is identified and analyzed. A lot of experiments have been done, and good results have been obtained. This shows that the new method of egg quality identification based on hyperspectral remote sensing image is feasible.
\end{abstract}

\section{INTRODUCTION}

The quality of eggs is divided into internal and external quality. Internal quality refers to egg protein quality, egg yolk quality and other indicators, such as odor, blood spots and chemical components. Protein quality indexes include protein height, Haugh unit and protein $\mathrm{PH}$ value. The quality of egg yolk mainly refers to the color of egg yolk and the strength of yolk membrane. The external quality includes eggshell strength, eggshell structure, eggshell color, egg weight and egg shape index. According to the quality index of eggs, the traditional detection methods are the sensory recognition and illumination recognition. With the increase in the consumption of eggs, it is very difficult for these traditional methods to meet the actual needs, especially the market supervision and management, and egg quality traceability. In recent years, with the continuous development of science and technology, some new technologies are constantly applied to egg quality detection, such as infrared spectroscopy [1], machine vision [2-4], computer image [5], dielectric property method [6], electronic nose [7, 8].

Hyperspectral imaging technology originated in 1980 s, it is mainly used to obtain the spectral characteristics of ground objects [9], and then gradually developed into archaeology, cultural relic identification and restoration, public safety and biometrics [10]. Especially in real and false recognition, true and false recognition and target classification, it has a wide range of applications. Because hyperspectral imaging can get the spectral characteristics of higher spectral resolution, making many works that are not recognized in panchromatic and multispectral data are very simple. This paper focuses on the detection of egg quality by using hyperspectral imaging technology, mainly studies the spectral characteristics of egg yolk, and performs classification and identification of egg quality with spectral characteristics of egg yolk. This new technology is very useful for the improvement of egg quality identification and the establishment of egg quality traceability system, and has important research value.

\section{EXPERIMENT DESIGN AND REALIZATION PROCESS}

The ground hyperspectral imager (MShyperSIS) was used to obtain the spectral characteristics of egg yolk. The spectral resolution of the system is $2.8 \mathrm{~nm}$, the imaging wavelength is $400 \mathrm{~nm}-1000 \mathrm{~nm}$, and the size of the obtained image is $1344 \times 1024$. Six different kinds of eggs were selected for comparative experiments, and ten eggs were selected for each type egg. The concrete experimental process is as follows.

(1) prepare different type eggs.

(2) The different eggs are numbered and marked, respectively.

(3) The hyperspectral imager is prepared and the machine is open.

(4) The eggs are got in sequence according to the order of different types of eggs, and the egg shells are broken, and the yolk and the egg white are effectively separated by the instrument. The separated yolk is put in an instrument or plate.

(5) Using the hyperspectral imager performs the scanned imaging to the yolk, and save the data.

(6) Using ENVI software to open the obtained hyperspectral remote sensing data and extract the spectral characteristics of egg yolk, and finally save them.

(7) Perform the filtering and normalization operations on the spectral data.

\section{DATA ACQUISITION AND ANALYSIS}

The spectral characteristics data of raw yolk is obtained by using hyperspectral imager, as shown in Figure 1 to Figure 4 . What Figure 1 shows is that the spectral data obtained by single egg yolk of each type egg. In other words, after the hyperspectral data cube of raw yolk is obtained, the acquisition of spectral data is measured by single egg yolk. The spectral data is obtained via mixing egg yolk of a certain type egg. That is, the hyperspectral image of the mixed egg yolk is obtained first, and then the spectral characteristic data is 
extracted from it. In Figure 1, when the obtained data is filtered, the size of the filter window is set to ten. The spectral characteristics of egg yolk which is shown in Figures 1 (a) - (f) shows is corresponds to from the first to the sixth different types of eggs, respectively. As you can see in Figure 1, that there are two spectral curves shown in Figure 1 (a) is looser and more discrepant than other. In Figure 1 (c), there are two singular values on the left. From the left end of the maximum peak, the spectral curves are different in Figures 1(d)-(f). The spectral characteristics data of all egg yolks of some type egg is treated by average, and then the curve of the approximate spectral characteristics of the egg yolk of this kind of egg

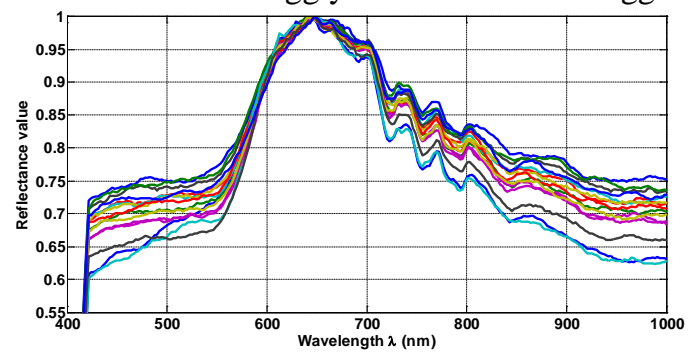

(a) The first type eggs

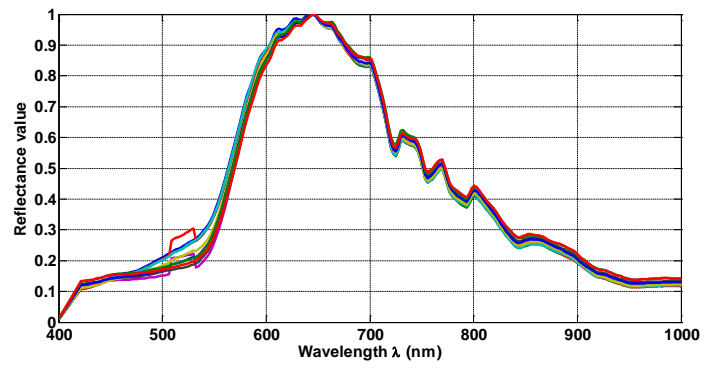

(c) The third type eggs

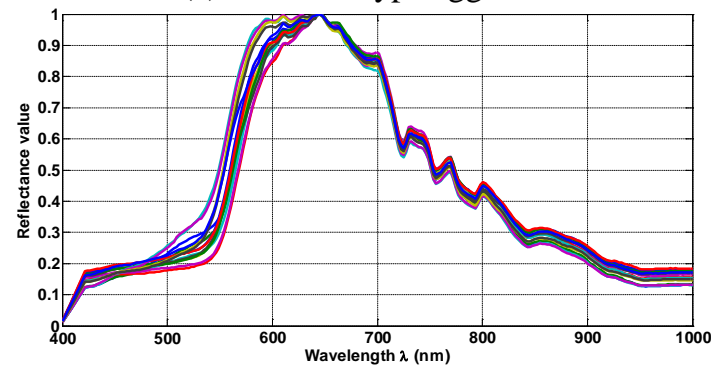

(e) The fifth type eggs is obtained, as shown in Figure 2. The data shown in Figure 3 is a reordering of spectral data shown in Figure 2. It can be seen in Figure 2 and Figure 3 that the spectral characteristics of egg yolks of the first and the second types of eggs are very close, and the curves between the third and the sixth types are very similar, also. This shows that the first and the second are the same type eggs, but the actual sign indicates that they are different types of eggs, indicating the possibility of imposing. And their storage time is longer than the other four types, that is, the freshness of them is poorer than other.

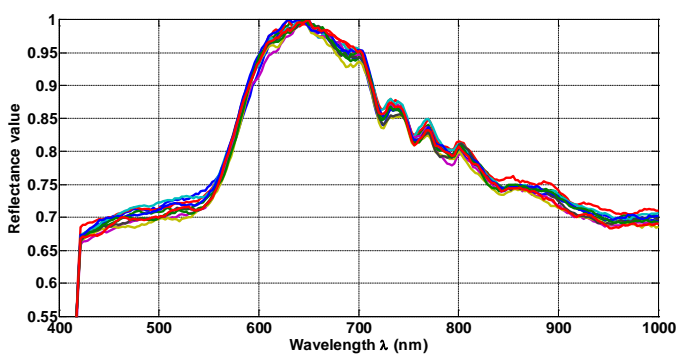

(b) The second type eggs

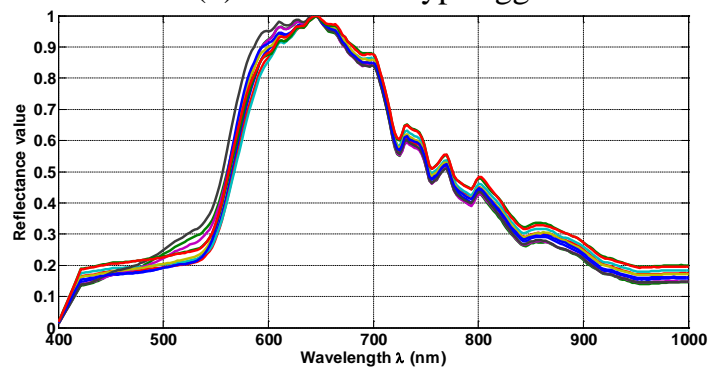

(d) The fourth type eggs

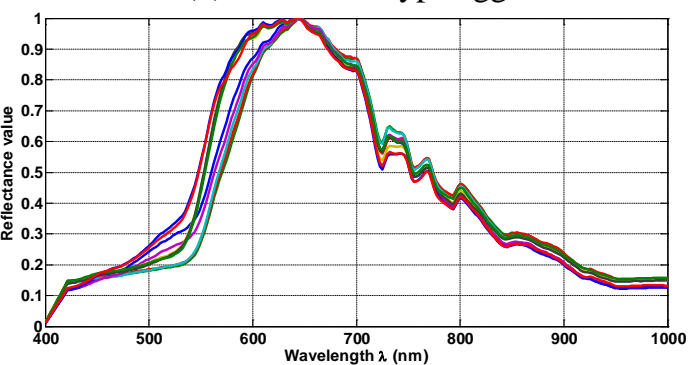

(f) The sixth type eggs

Figure 1 Spectral characteristic curves of egg yolk from different eggs

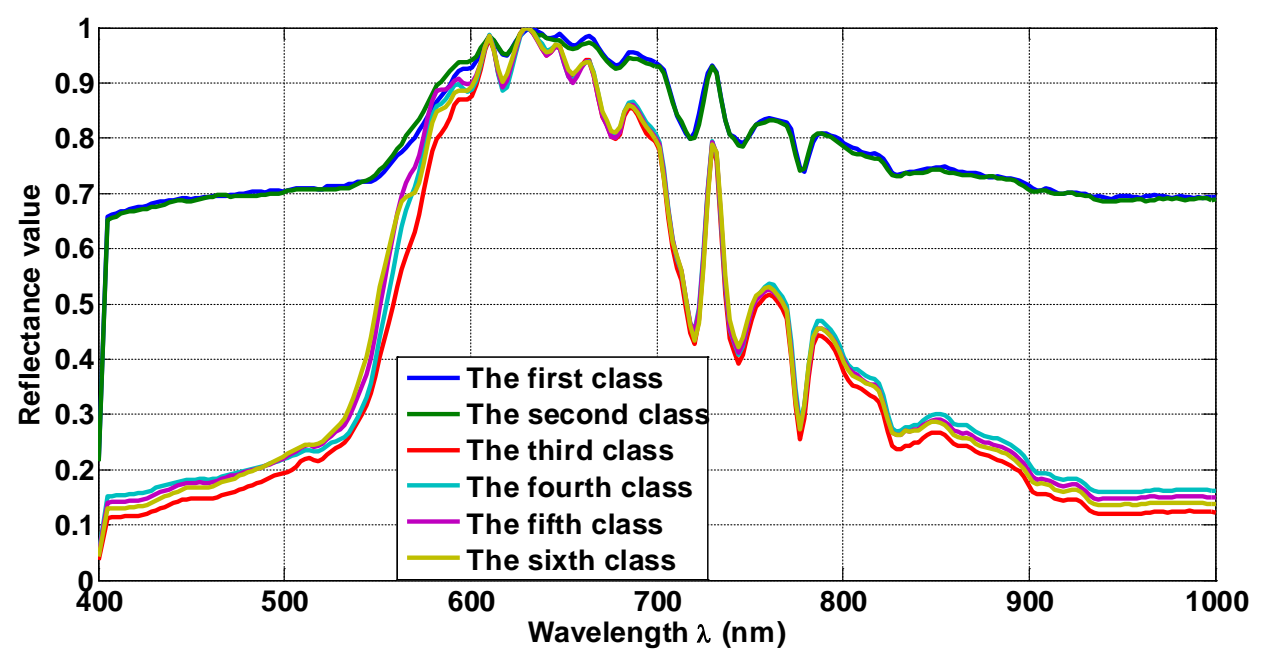

Figure 2 The average spectral characteristic curves of egg yolk of different type eggs 


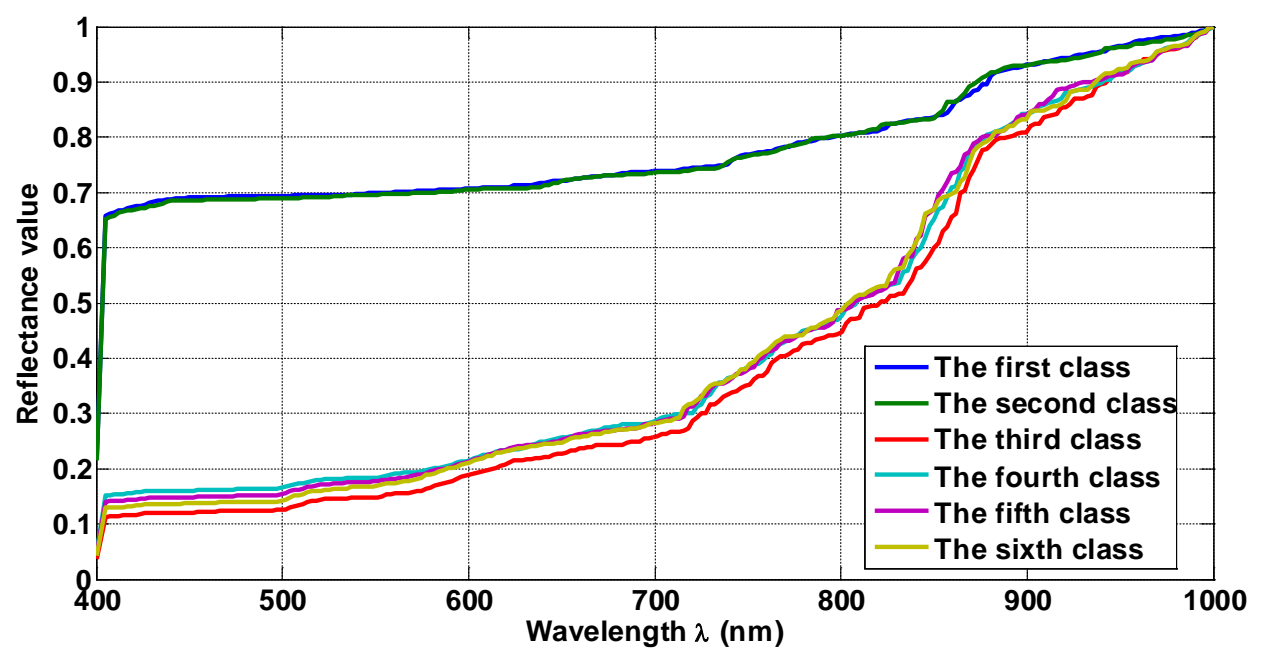

Figure 3 Reordering curves of average spectral characteristic data

The measurement of the spectral characteristics of a mixture of egg yolk is shown in Figure 4. It can be seen in Figure 4, in the data shown in Figure 4 (a), the spectral values extracted at different measuring points are different, and a measured value in Figure 4 (b) has a small range of differences. The mixed value of other types of egg yolk is normal, especially the sixth type of egg. This shows that the egg yolk of the sixth types of eggs is almost the same; the egg yolk components of the first type to the sixth type of eggs have some differences.

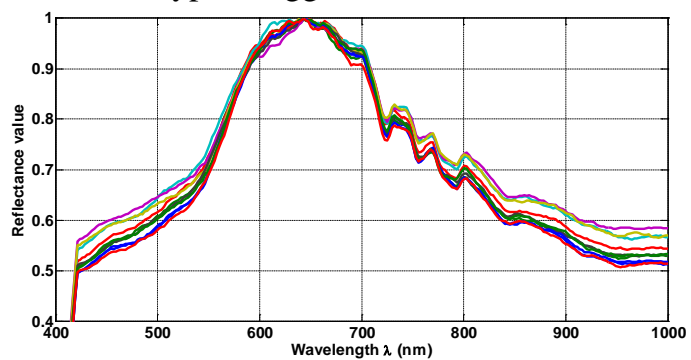

(a) The first type eggs

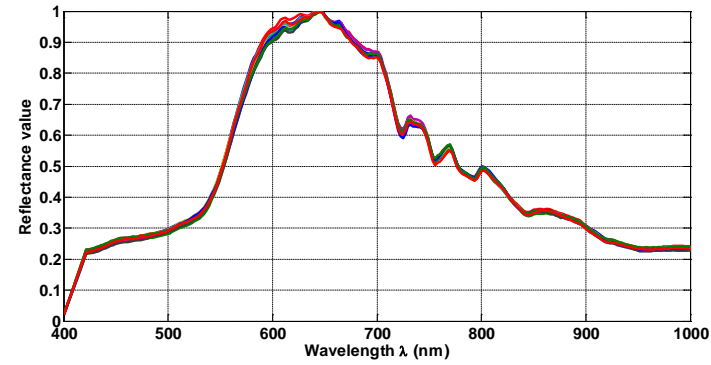

(c) The third type eggs

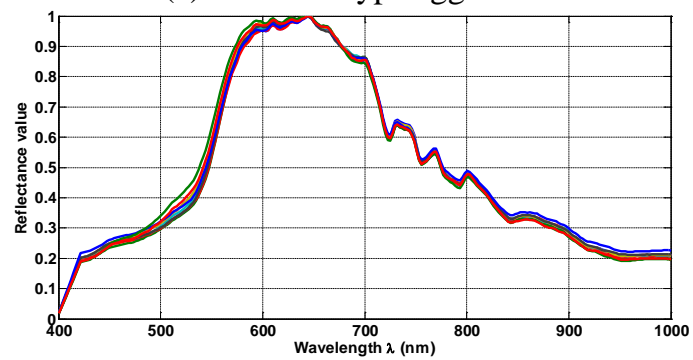

(e) The fifth type eggs
The average spectral characteristic value of the mixed egg yolk is measured and shown in Figure 5. In the spectral characteristics shown in Figure 5, the first type egg is very different from other types of eggs in spectral characteristics. This shows that the first type of egg has a long retention time, close to the state of egg yolk powder yellow, and the freshness is very poor. The sixth type of eggs is a completely natural breeding free range egg, so after mixing different eggs, there is almost no difference among the egg yolk.

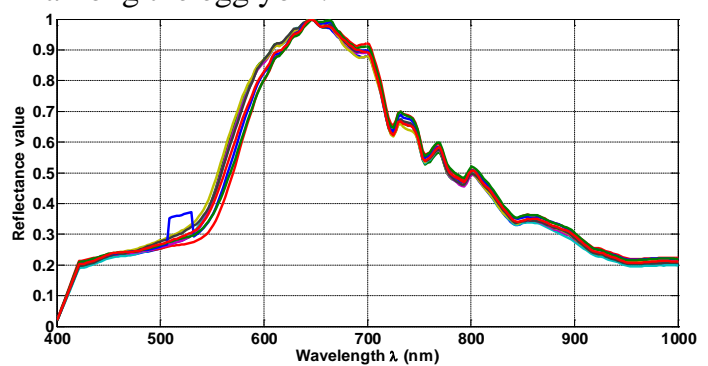

(b) The second type eggs

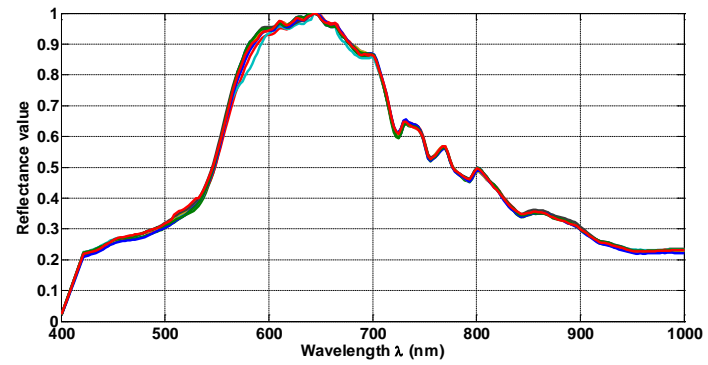

(d) The fourth type eggs

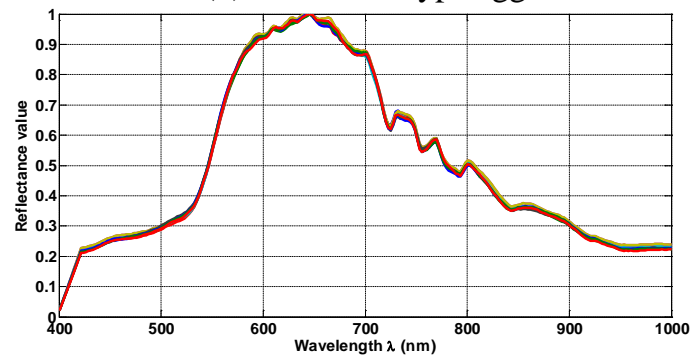

(f) The sixth type eggs

Figure 4 The spectral characteristics of mixed egg yolk of different type eggs 


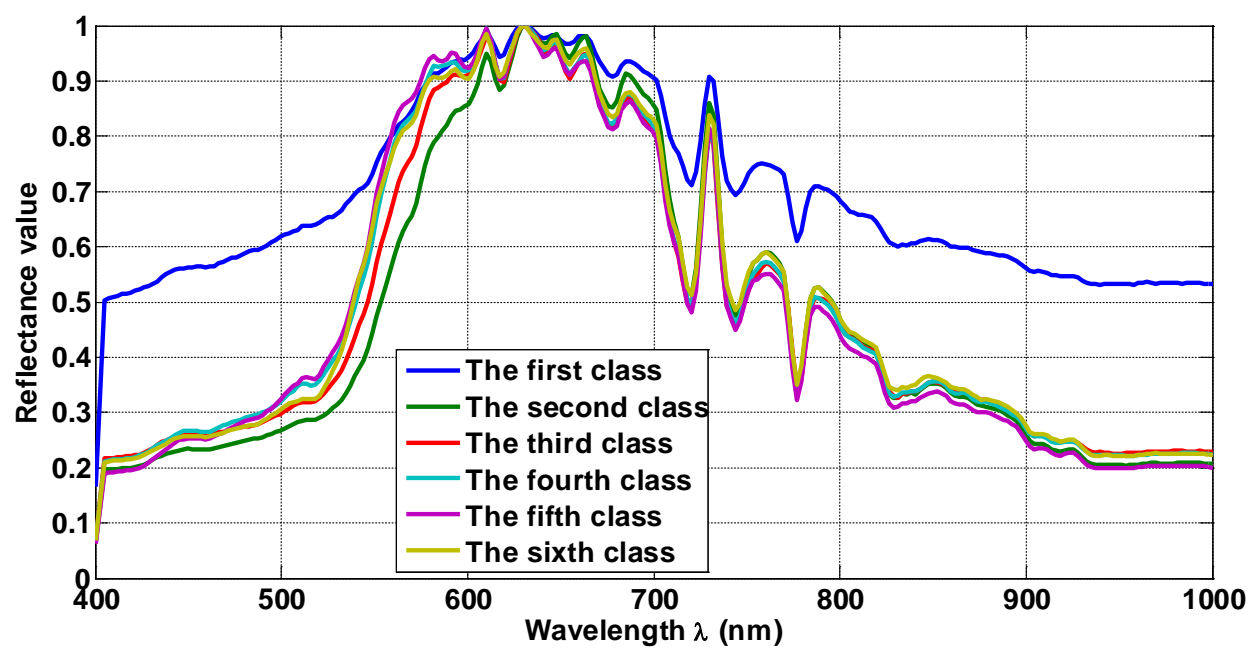

Figure 5 The average spectral characteristic curves after mixing egg yolk

\section{CONCLUSIONS}

Through the exploration and practice of this paper, it is shown that hyperspectral imager can be used to study the spectral characteristics of egg yolk. The experimental results show that the spectral characteristics of egg yolk based on hyperspectral images can effectively detect the freshness of eggs and the differences of egg yolk components. The difference in egg yolk components is caused by different feeding methods and different feed, so it provides a new way of thinking for the establishment of egg quality traceability system.

\section{ACKNOWLEDGEMENTS}

The work was supported by Natural Science Foundation of China (No. 41574008, 61379031), Natural Science Basic Research Plan in Shaanxi Province of China (No. 2016JM6052) and Special Foundation for Special Talents of Xijing University (No. XJ17T04).

\section{REFERENCES}

[1] Nicolas A N, Michael N, Shiv P, Yousef K. Prediction of egg freshness and albumen quality using visible/near infrared spectroscopy. Food and Bioprocess Technology, 2011, 4(5):731-736.

[2] Omid M, Soltani M, Dehrouyeh M H, et al. An expert egg grading system based on machine vision and artificial intelligence techniques. Journal of food engineering, 2013, 118: 70-77.

[3] Duan Y F, Wang Q H, Li X M, Tang Y H. High-throughput on line detection method of egg size and shape based on convex hull algorithm. Transactions of the Chinese Society of Agricultural Engineering, 2016, 32(15):282-288.

[4] Abdullah M H, Nashat S, Anwar S A, Abdullah M Z. A framework for crack detection of fresh poultry eggs at visible radiation. Computers and Electronics in Agriculture, 2017, 141, 81-95.

[5] Priyadumkol J, Kittichaikarn C, Thainimit S. Crack detection on unwashed eggs using image processing. Journal of Food Engineering, 2017, 209, 76-82.

[6] Sun J, Liu B, Mao H P, et al. Non-destructive examination for freshness of eggs based on dielectric properties and yolk index regression model. Transactions of the Chinese Society of Agricultural Engineering, 2016, 32(21): 290 -295.

[7] Li J T, Zhu S S, Jiang S, Wang J. Prediction of egg storage time and yolk index based on electronic nose combined with chemometric methods. LWT - Food Science and Technology, 2017, 82, 369-376.

[8] Wang Q H, Zhou K, Wang C Y, et a1. Egg freshness detection based on hyperspectral image technology. Advance Journal of Food Science and Technology, 2015, 7(8): 652-657. [9] Xia J, Yokoya N, Iwasaki A. Hyperspectral image classification with canonical correlation forests. IEEE Transactions on Geoscience and Remote Sensing, 2017, 55(1): 421-431.

[10] Lowe A, Harrison N, French A P. Hyperspectral image analysis techniques for the detection and classification of the early onset of plant disease and stress. Plant methods, 2017, 13(1): 80. 